\title{
INVESTIGATION OF SOME OXAZOLONE DERIVATIVES AS ULTRAVIOLET ABSORBERS IN COMMERCIAL CHEMICAL COMPOSITIONS
}

\author{
M.SH.FAYED*
}

\begin{abstract}
:
Solar radiation consists of a continuous spectrum of wavelengths ranging from infrared through visible light to the ultraviolet radiation region. The latter represents a challenge to certain commercial chemical compositions such as exterior paints, plastics, sunscreen cosmetics and others. Ultraviolet absorbers are such compounds added to the compositions in small percentage to protect them against their deterioration by UV radiation. Some oxazolone derivatives were prepared, purified and tested as UV absorbers as $0.02-2 \%$ solutions in organic solvents. The solvents used were: ethanol, toluene, acetone, benzyl alcohol, acetonitrile, THF, n-butanol and 1,4-dioxan. The transmittance was measured in the range of 200-400 $\mathrm{nm}$. The effect of oxazolone derivative type and concentration in the given solvents were shown and discussed. The transmittance ranging between $0.3-1.9 \%$ of UV radiation in the range of $260-400$ $\mathrm{nm}$, depending on the solution.
\end{abstract}




\section{INTRODUCTION:}

Light in the UV region has an appropriate amount of energy to cleave chemical bonds in some products. This cleavage produces free radicals which must be prevented by the absorbtion of such fraction of light before an undesirable chain reaction starts. The absorbtion is accomplished by apecific chemical compounds called UV absorbers, added to the commercial composition in small percentage.

In the field of plastics, the reported four major classes of UV absorbers were derivatives of hydroxy benzophenone, hydroxy benzotriazole, diaryl terphthalate and cinnamic acid [1]. Sometimes certain derivatives of tetramethylpyridine were also used [2].

In the domain of exterior paints, substituted benzophenones or benzotraizole type were added as UV absorbers [3]. They should be matched to the used particular resin so that it can be protected from the UV wavelengths to which it is most sensitive [4].

In the cosmetics field several classes were used as sunscreens and are classified into sunburn preventive, suntanning or opaque sunblock agents depending on the wavelengths absorbed and the degree of absorption [5].

In this work, 4-arylidene-2-phenyl-5-oxazolone derivatives were prepared and tested in solution, as UV absorbers. The testing was done by the measuement of transmitted UV light in the range of 200-400 $\mathrm{nm}$.

\section{EXPERIMENTAL:}

Preparation of Oxazolone Derivatives [6]:

An intimate mixture of the aromatic aldehyde( $30 \mathrm{mmol})$, hippuric acid (30 $\mathrm{mmol})$, anhydrous sodium acetate $(30 \mathrm{mmol})$ and acetic anhydride $(20 \mathrm{ml})$ was prepared in 100-ml conical flask over a hot plate until the reaction mixture became completely molten. The bright yellow to pale brown liquid was heated at about 95-100 C for two hours, then cooled and 30ml ethanol was added. The mixture was again heated at about $95-100 \mathrm{C}$ for 0.5 hour to convert the acetic anhydride to ethylacetate. The mixture was cooled, and the product was 
filtered, washed successively with boiling water $(2 \times 50 \mathrm{ml})$ and cold ethanol $(2 \times 50 \mathrm{ml})$. The product was recrystallised from benzene to give a yellow crystals of constant melting point:

4-bezylidene-2-phenyl-5-oxazolone: yield 56\%; m.p. 166-167 C

4-furfurylidene-2-phenyl-5-oxazolone: yield 52\%; m.p. 158-160 C.

All chemicals used in the preparation were of laboratory grade and are directly used without further treatment.

The transmittance of UV radiation was measured by Shimadzu UV-Visible recording spectrophotometer, UV-160. The range of measurements was from 200-400 $\mathrm{nm}$. The derivative was dissolved in different solvents with different concentrations. The solvents used were acetone, ethanol, tetrahydrofuran, acetonitrile, toluene, benzyl alcohol, acetone, n-butyl alcohol, dioxan and n-hexane.

\section{RESULTS AND DISCUSSION:}

aldehydes readily condense with hippuric acid in the presence of acetic anhydride to yield 2,5-disubstituted-5-oxazolones (azlactones). The reaction propably involves the cyclization of the hippuric acid to the 5-oxazolone followed by the condensation of the selected aldehyde with the active methylene group at the position 4 to produce the azlactones. These compounds were selected to be tested as UV absorbers.

The main and necessary property for the UV absorber is to be effective in absorbing the UV radiation in the range characterized by the desired application. In sunscreen agents this range is reviewed to be from $290 \mathrm{~nm}$ to $320 \mathrm{~nm}$. It can be sunburn preventive if it absorbs $95 \%$ of UV radiation in this range or suntanning if it absorbs at least 85\% [5]. The opaque sunblock agents provide maximum protection in the form of physical barrier (through reflection and scattering) to all radiation in the whole range from $290 \mathrm{~nm}$ to $700 \mathrm{~nm}$.

In paint technology, the amount of various UV absorbers which can be employed depends upon the degree of stability desired, the amount of harmful residues in the polymer to be stabilized and the surrounding conditions. It is considered that about $5 \%$ by weight would be the maximum required for preventing degradation of the polymers under severe conditions[7]. In some instances as little as $0.1 \%$ of each stabilizing additive will be adequate to prevent visible and UV light degradation. Generally, it was acceptable in literature that any one of the additives should not be used in amount greater than ten times the weight of any other additive that may be present. 
The prepared 4-benzylidene-2-phenyl-5-oxazolone was dissolved in different solvents with different concentrations and tested as UV absorber in the range of $200 \mathrm{~nm}$ to $400 \mathrm{~nm}$. The obtained results is shown in Table 1. It is clear that the mentioned compound absorbs the UV radiation by an amount ranging between $98 \%$ to more than $99.5 \%$ in the two bands UV-A (from 320-400 nm) and UV-B (from 290-320 $\mathrm{nm}$ ) and with little effect on the band UV-C which is the germicidal range $(200-290 \mathrm{~nm})$. The effectiveness in the harmful range of UV radiation (290-320 $\mathrm{nm}$ ) commonly known as middle range depended on the nature of the organic solvent.

In toluene, acetone, acetonitrile and benzyl alcohol, the effectiveness was shifted to longer wavelenghths starting at $310-380 \mathrm{~nm}$ according to the solvent. In the case of 1,4-dioxan, ethanol and tetrahydrofuran, the shift was to the shorter wavelengths starting at about 240 or $260 \mathrm{~nm}$ and the effectiveness was expanded to the two bands of UV-A an UV-B. So the effectiveness of the compound was influenced much by the nature of solvent and to a little extent by the tested concentration range from 0.01 to $2 \%$ solution.

According to these results, this oxazolone derivative can safely be used in the field of plastics and exterior paints but in the field of cosmetics it absorbs much of the UV radiation in the range of $320 \mathrm{~nm}$ to $400 \mathrm{~nm}$ that is responsible for the maximum skin tanning.

In the case of 4-furfurylidene-2-phenyl-5-oxazolone, two solvents were chosen for the comparison: ethyl alcohol and 1,4-dioxan, shown in Table 2.

In alcoholic solution, a complete UV absorption occurs in the range of 240-400 $\mathrm{nm}$ with concentrations starting from $0.02 \%$ to $0.52 \%$. With excess dilution and starting from $0.01 \%$, there appeared a region of higher transmittance from $280-350 \mathrm{~nm}$ with a maximum of $8 \%$ augmented to $20 \%$ at $0.001 \%$ solution at 320 $\mathrm{nm}$.

The same features occured in case of $0.004 \%$ in 1,4-dioxan solution, but with about 28\% maximum tranmittance at $320 \mathrm{~nm}$. complete absorption of UV occured starting from 350 to $400 \mathrm{~nm}$.

From such results solutions of $0.02-2 \%$ could act as sunburn preventive in the $U V-A$ and UV-B bands. But with lower concentration their effect remains mainly in the UV-A band only, the range responsible for the maximum skin tanning and with a variable effect in the UV-B band depending on the type of the the oxazolone derivative and its concentration. 
On the other hand, these oxazolone derivatives are colourless compounds, so they can be employed with the assurance that they will not contribute to colour in normally colourless formulations, nor will they affect the colour of coloured formulation such as a paint film, a dyed textile or a plastic component. In the same time, these organic oxazolone derivatives will be easily homogenized with the main polymeric substrate having the same nature.

\section{CONCLUSION:}

The prepared 4-arylidene-2-phenyl-5-oxazolone derivatives proved to be good UV absorbers in solution in the range from 240-400 $\mathrm{nm}$. The effective concentration range was from $0.02-2 \%$. They can be widely used in the fields of plastics and exterior paints but in the field of cosmetics they highly affected the band of skin tanning. This investigation is an initial qualitative examination before commercial application.

\section{REFERENCES:}

1.Desk-Top Data Bank,"Additives and Plastics",1st Ed.,Laumann (1987).

2.Mascia,L.,"The Role of Additives in Plastics",Edward Arnold (1974).

3.Morgans,W.M.,"Outlines of Paint Technology",Vol.2,John Wiley (1984).

4.Oil and Colour Chemists Association, Australia,"Surface Coatings",Vol.2, Chapman and Hall (1984).

5.Wilkinson,J.B. and Moore,R.J., "Harry's Cosmeticology", 7th Ed., Longmam Scientific and Technical (1987).

6.Fitton and Smalley,"Practical Heterocyclic Chemistry", John Wiley (1972).

7.Rothenberg,G.B., "Paint Additives, Recent Developments", Noyes Data Corporation (1978). 
Table 1 The Effect of 4-benzylidene-2-phenyl-5-oxazolone as UV absorber in Different Solvents with Different Concentrations

\begin{tabular}{|c|c|c|c|}
\hline Solvent & $\begin{array}{c}\text { C } \\
\text { Concentration } \\
\text { Range (\%) }\end{array}$ & $\begin{array}{c}\text { Transmittance } \\
(\%)\end{array}$ & $\begin{array}{c}\text { Wavelength } \\
(\mathrm{nm})\end{array}$ \\
Toluene & $0.02-2$ & $0.3-1.9$ & $310-400$ \\
Acetonitrile & $0.015-1.5$ & $0.3-3$ & $340-380$ \\
Acetone & $0.028-0.25$ & $0.3-0.9$ & $330-400$ \\
1,4-dioxan & $0.042-0.5$ & $0.3-0.4$ & $260-400$ \\
Benzyl alcohol & $0.02-0.125$ & $0.3-0.5$ & $380-400$ \\
Tetrahydrofuran & 0.2 & $0.3-0.8$ & $260-400$ \\
Ethyl alcohol & $0.04-0.02$ & 0.3 & $240-390$ \\
\hline
\end{tabular}

Table 2 The Effect of 4-furfurylidene-2-phenyl-5- oxazolone as UV absorber

\begin{tabular}{|c|c|c|c|}
\hline Solvent & $\begin{array}{c}\text { Concentration } \\
\text { Range (\%) }\end{array}$ & $\begin{array}{c}\text { Transmittance } \\
(\%)\end{array}$ & $\begin{array}{c}\text { Wavelength } \\
(\mathrm{nm})\end{array}$ \\
Ethyl alcohol & $0.02-0.52$ & 0.3 & $240-400$ \\
1,4-Dioxan & $0.02-0.2$ & 0.3 & $260-400$ \\
\hline
\end{tabular}

